OPEN

SUBJECT AREAS:

SELF-ASSEMBLY

COLLOIDS

NANOPARTICLES

NANOFLUIDICS

Received

22 May 2013

Accepted

19 June 2013

Published

9 July 2013

\section{Correspondence and} requests for materials should be addressed to H.F. (frusawa.hiroshi@ kochi-tech.ac.jp)

\title{
Electric moulding of dispersed lipid nanotubes into a nanofluidic device
}

\author{
Hiroshi Frusawa' , Tatsuhiko Manabe' , Eri Kagiyama' ', Ken Hirano ${ }^{2}$, Naohiro Kameta $^{3}$, Mitsutoshi Masuda ${ }^{3}$ \\ \& Toshimi Shimizu ${ }^{4}$
}

\begin{abstract}
${ }^{1}$ Institute for Nanotechnology, Kochi University of Technology, Tosa-Yamada, Kochi 782-8502, Japan, ${ }^{2}$ Health Research Institute, National Institute of Advanced Industrial Science and Technology (AIST), 22 17-14, Hayashi-cho, Takamatsu, Kagawa 761-0395, Japan, ${ }^{3}$ Nanosystem Research Institute, National Institute of Advanced Industrial Science and Technology (AIST), Tsukuba Central 5, 1-1-1 Higashi, Tsukuba, Ibaraki 305-8565, Japan, ${ }^{4}$ AIST Fellow, National Institute of Advanced Industrial Science and Technology (AIST), Tsukuba Central 5, 1-1-1 Higashi, Tsukuba, Ibaraki 305-8565, Japan.
\end{abstract}

Hydrophilic nanotubes formed by lipid molecules have potential applications as platforms for chemical or biological events occurring in an attolitre volume inside a hollow cylinder. Here, we have integrated the lipid nanotubes (LNTs) by applying an AC electric field via plug-in electrode needles placed above a substrate. The off-chip assembly method has the on-demand adjustability of an electrode configuration, enabling the dispersed LNT to be electrically moulded into a separate film of parallel LNT arrays in one-step. The fluorescence resonance energy transfer technique as well as the digital microscopy visualised the overall filling of gold nanoparticles up to the inner capacity of an LNT film by capillary action, thereby showing the potential of this flexible film for use as a high-throughput nanofluidic device where not only is the endo-signalling and product in each LNT multiplied but also the encapsulated objects are efficiently transported and reacted.

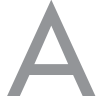

large research effort has been made to organise colloidal particles into functional materials, and we now have a variety of elaborate techniques for engineering colloids including functional nanoparticles ${ }^{1-6}$. Colloidal methods guide particle configurations in desired directions using the bottom-up phenomena of either self-organisation ${ }^{1-5}$ or directed assembly using external fields ${ }^{3-6}$. Polymerized crystalline arrays of spherical colloids represent the bottom-up assembly into macroscopic or mesoscopic materials, which has been found to be available for use in photonic devices ${ }^{1,2,7}$. Furthermore, the assembly materials serve for the efficient utilisation of the constituents' individual properties.

Among the functional nanoparticles of various shapes, we focus on lipid nanotubes (LNTs) whose tubular structures include those with hydrophilic internal and external membrane surfaces formed by lipid molecules ${ }^{8-16}$. The LNT used has a typical $10-\mathrm{nm}$ inner diameter and $10-\mu \mathrm{m} \mathrm{length}^{12-16}$, and therefore provides the confined liquid space of ten attolitre volume, which is smaller than the volumes of a femtolitre chamber and a single cell by factors of $10^{2}$ and $10^{5}$, respectively ${ }^{13,14}$. The confined liquid nanospace has found potential in chemical and biological applications of LNTs as well as polymer nanotubes ${ }^{16-22}$, including controlled drug release ${ }^{18-21}$ and artificial chaperoning of denaturated proteins ${ }^{22}$. An individual LNT has thus been found to be useful as a nanoreactor and/or nano-assay device ${ }^{23-28}$. Correspondingly, a variety of techniques has been developed for manipulating and integrating LNTs into ordered nanocapillary arrays ${ }^{29-36}$.

Despite the numerous attempts, there remain unresolved issues in creating nanofluidic devices of LNT arrays $^{21,34-41}$. Here we address the methodological challenges associated with the following: (i) sufficient total output, (ii) efficient transport and reaction inside the hollow cylinder, and (iii) no addition of binder molecules to keep the native outer surfaces.

(i) First, the increase in the total output requires the cost-effective assembly of a considerable number of nanocapillaries into the oriented arrays. We need $10^{5} \mathrm{LNT}$ s for the output to be equivalent to that of a single cell. Therefore, it is natural to highlight the bottom-up assembly techniques instead of the top-down approaches. (ii) Second, a high-throughput device requires that open ends are connected by a seamless nanocapillary inside a single LNT (see Fig. 1(a)), which is similar to the ideal state of semiconducting carbon nanotubes in creating a thin film transistor ${ }^{42,43}$. This bridging can be accomplished by excluding serial arrays of LNTs from the oriented assembly. (iii) Last, a genuine assembly with an unaltered native outer surface is vital to the straightforward scaleup of a pilot study on a single LNT, a nano-platform for reacting and transporting encapsulated objects. 
a

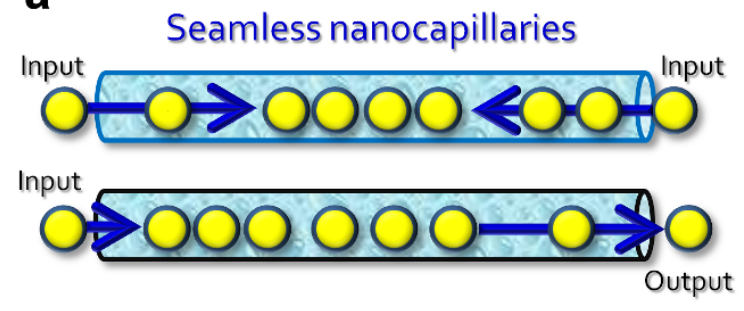

Grafted nanocapillaries

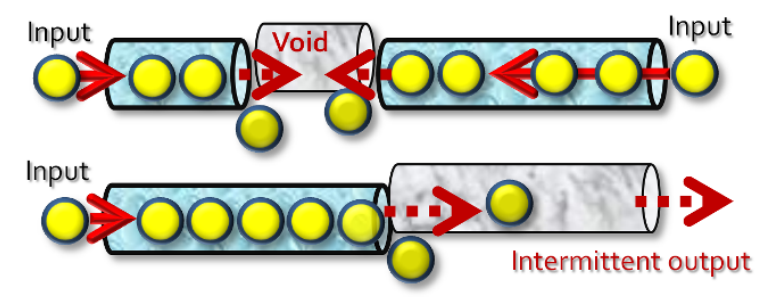

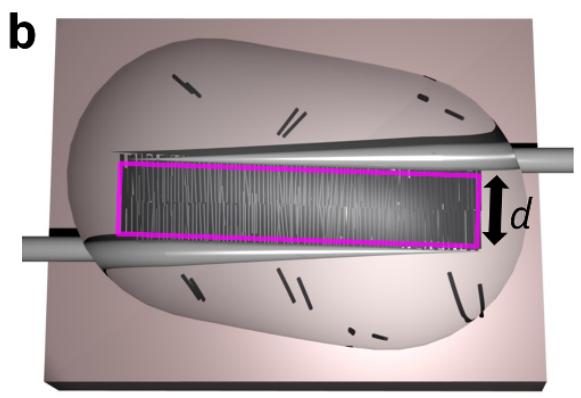

C

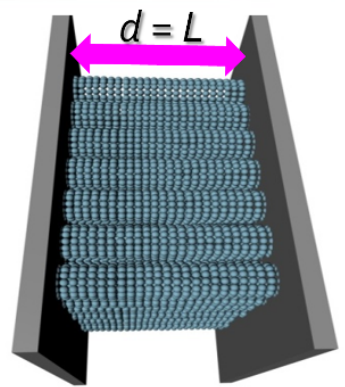

Figure $1 \mid$ Off-chip assembly of dispersed LNTs into parallel nanocapillary arrays. (a) Comparison between seamless and grafted nanocapillaries. The upper situation illustrated by two schematics represents seamless nanocapillaries that have high-throughput processing. The lower diagrams correspond to instances of inefficient transportations and reactions, which demonstrates that the graft-induced jamming of injected particles either stops the flows out of the nanotubes or causes incomplete immersion of the inner space, leaving void nanotubes. (b) Illustration of the plug-in system. An external AC electric field is locally applied to a drop of an LNT suspension by inserting a pair of electrode needles controlled by patch-clamp micromanipulators into the solution. The purple rectangle marks the region for fundamental studies of the assembly processes. (c) Schematic representation of parallel nanoapillary arrays that are formed by sandwiched LNTs when the electrode gap $d$ is adjusted to the mean long axial length $L$ of a single LNT.

\section{Results}

Off-chip assembly for creating nanofluidic devices. In order to fabricate a flexible nanofluidic device of LNTs fulfilling the aforementioned requirements, we exploited an AC electric field to direct the organisation of nanoparticles $s^{4-6,42-50}$. While previous studies on the electric assembly of colloidal spheres have demonstrated that AC fields applied via on-chip electrodes precisely control the 3D and 2D crystalline orientations as well as the colloidal concentrations $s^{4-6,44-48}$, on-chip assembly systems have problems arising from the electrodes being fixed on a substrate. Because the electrode configuration cannot be changed, on-chip systems are irrelevant to electrode removal from a colloidal assembly without the use of a binder. Additionally, on-chip processes depend on the wettability of the substrates and limit the substrates that can be used for applications.

To overcome these problems, we adopt the off-chip assembly system that plugs into a sample drop a pair of electrode needles above a cover slip (see Fig. 1(b)). The rectangle delineated in Figure 1(b) corresponds to an off-chip potential valley created by the needle pair. Because the plug-in electrodes are independently controlled by two sets of patch clamp micromanipulators, the off-chip system facilitates to adjust the electrode gap so that serial nanocapillary alignments may be excluded, thereby creating an LNT film of parallel nanocapillary arrays (see Fig. 1(c)). Moreover, the off-chip style has advantages of avoiding both impurity deposition and dryinginduced adhesion to either the electrodes or the substrate. Therefore, we are able not only to control the assembly size, but also to remove an assembly from the needles, which yields a separated genuine assembly of an appropriate size. We can see the assembly and drying sequences for the LNT suspension in Supplementary Movies 1 and 2, respectively.

The LNT length distribution has been efficiently determined using in situ measurements of the rotational dynamics in the LNT suspension (see Supplementary Discussion for the details) ${ }^{33}$. The histogram in Supplementary Figure S1 shows that the majority of the lengths are between four and six microns; the mean length is calculated to be approximately $6 \mu \mathrm{m}$. It is also to be noted that the off-chip assembly was performed in a filtered dispersion where shorter LNTs had passed away through a $5-\mu \mathrm{m}$ plastic filter (see Methods). Accordingly, an electrode gap less than $10 \mu \mathrm{m}$ can considerably diminish the number of serial-parallel arrays, the alignment of two or more LNTs along the external electric field, so that we can obtain an LNT film whose majority is formed by parallel nanocapillaries. In what follows, we demonstrate that this off-chip technique using plug-in electrodes allows one-step fabrication of biocompatible nanofluidic devices solely using LNTs, which will be referred to as "electric moulding".

Electric moulding into birefringent LNT films. Figure 2(a) exhibits three typical images of LNT films. A digital microscope image on the upside shows a spontaneously dried film on a cover glass, a substrate used in separating LNT assembly from manipulated electrodes (the last procedure of electric moulding). While the LNT film in the upper image, which leaves moist, has a $20-\mu \mathrm{m}$ width and $70-\mu \mathrm{m}$ length in accordance with the electrode configuration, it is possible to change the size of the electrically focused area into $10-\mu \mathrm{m}$ in gap length which satisfies the aforementioned requirement of parallel LNT arrays. The lower images in Figure 2(a) represent the narrower LNT films having $10-\mu \mathrm{m}$ width. These films are placed on a grid substrate to perform in situ observations using scanning electron microscopy (SEM) as shown in the lower right image, and the metallic substrate can be seen through a transparent LNT film in the digital microscope image on the lower left side.

Electrode removal does not always succeed, and the left micrograph in Figure 2(b) demonstrates a typical tearing situation during the separation process from the electrodes; one of the methodological refinements for reducing incomplete removal would be to improve the electrode surface using chemical modification. At the same time, the left image suggests that each individual LNT is perpendicular to the surface of electrode needles, which has been verified by the middle SEM image in Figure 2(b). The SEM image focuses on an edge parallel to the electrode surface, showing the alignment of LNTs. The polarised optical micrograph on the rightmost side of Figure 2(b) also demonstrates the birefringent property of LNT films placed on 
a

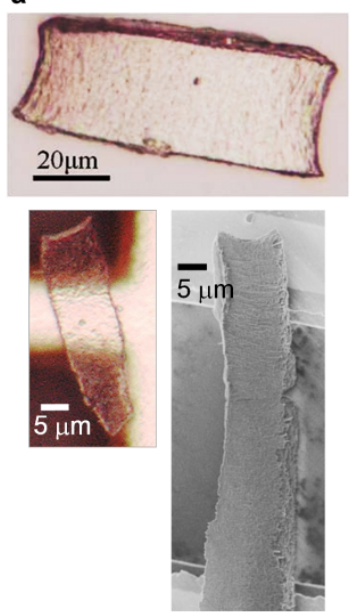

b

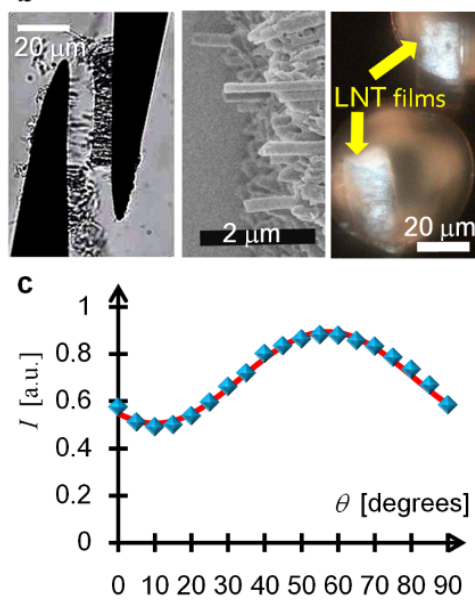

Figure $2 \mid$ Anisotropy of electrically moulded LNT films. (a) A digital microscope image on the upside shows a film of LNT arrays successfully removed from the electric mould with a width and length of $20 \mu \mathrm{m}$ and $70 \mu \mathrm{m}$, respectively. The lower images, on the other hand, represent narrower LNT films having a width of approximately $10 \mu \mathrm{m}$. These LNT films are placed on a metallic grid substrate, on which in situ observations are performed using digital microscopy (left image) and SEM (right image). (b) The leftmost micrograph shows an instant the needles were removed from the dried assembly. The middle SEM image provides a magnified view of an edge, which verifies that LNTs are aligned in the desired direction. The birefringence due to the LNT alignment is seen from the right image, the polarised optical micrograph using cross Nicols, where we can find a pair of bright LNT films on the SEM grid. (c) The blue squares show the angular dependence of the mean brightness evaluated using polarised microscopy when the sample stage is successively rotated. The red sine curve is fitted to the data.

the SEM grid. The film brightness measured in the polarised micrograph varies when the stage is rotated. Image analysis provides the intensity of the transmitted light, normalised by the maximum, which is then averaged over the regions of these films to obtain a

mean brightness $I(\theta)$ as a function of the rotation angle $\theta$ of the stage (see Fig. 2(c)). The fitting result is given by $I(\theta)=A_{0}+A \sin \{2 \pi(\theta)$ $\Theta)+\delta\}$ with a set of optimized parameters, $A_{0}=0.7, A=0.19, \Theta=$ $90^{\circ}$, and $\delta=-129^{\circ}$, quantifying the anisotropic optical property common to liquid crystalline materials.

Size-selective inclusion of GNPs into LNT films. The birefringent film of aligned LNTs is expected to selectively and efficiently capture aqueous nanoparticles by capillary action, when the target film is dried in a vacuum. We demonstrate below that the LNT films fabricated by our electric moulding technique hold this capability of individual LNTs, using both the digital microscopy and the fluorescence resonance energy transfer (FRET) technique. It has been found that the FRET solely occurs from the internal fluorescent molecules of Rhodamine 6G (R6G) to GNPs encapsulated in the inner space of LNTs because the thickness of the LNT membrane wall is approximately $80 \mathrm{~nm}$ and is large enough to prevent FRET through the wall ${ }^{23}$; therefore, the FRET is relevant to the detection of nanoparticle encapsulation inside the LNTs.

The use of 5-nm GNPs provides the digital microscope images of Figure 3(a) where we observed that the LNT films were kept metallic after rinsing GNPs repeatedly, which is similar to the micrographic look of metallic grid in Figure 2(a). Correspondingly, the addition of R6G molecules mostly exhibits no fluorescence due to the FRET, or the disappearance of the fluorescence band, and a fluorescence micrograph on the right side of Figure 3(a) selects an incomplete film that is somehow recognizable due to a partially fluorescent part where the lack of GNPs results in the irrelevance of the FRET. As seen from the digital microscopy image of Figure 3(b), on the other hand, an LNT film mixed with 40-nm GNPs exhibits a normal surface indistinguishable from that of a genuine LNT film, which conforms with a fluorescent brightness highlighting the outline in the fluorescence micrograph on the right side of Figure $3(\mathrm{~b})$. The entire absence of FRET in adding 40-nm GNPs offers some explanations as follows: while most of 40-nm GNPs are excluded from the hollow channels of LNTs having its inner diameter of $40 \pm 10 \mathrm{~nm}$, the saline rinses serve to eliminate GNPs coated through vacuum drying and the FRET on the surface is negligible even if the covered GNPs are left to some extent.
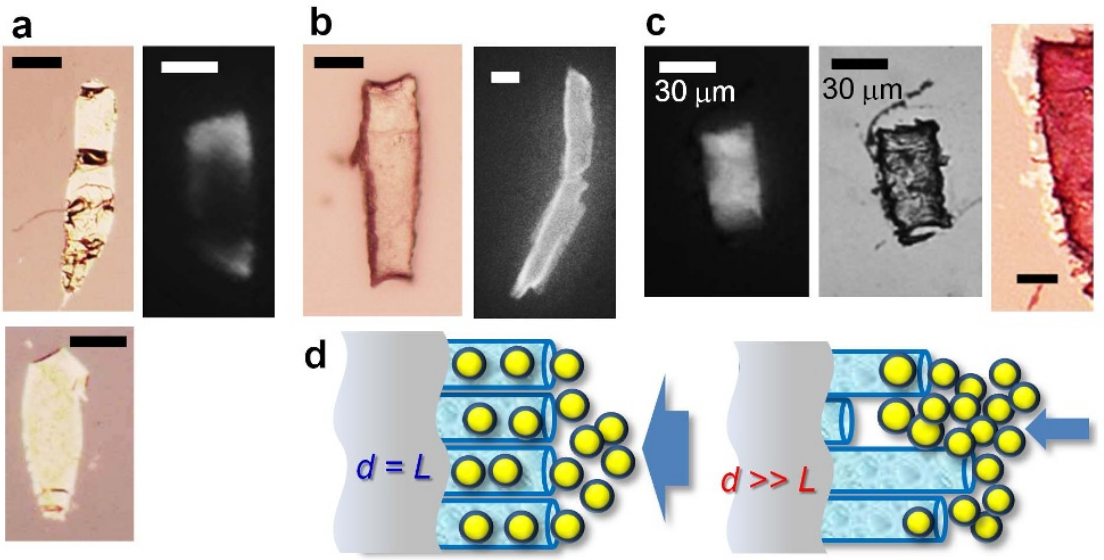

Figure 3 Addition of gold nanoparticles to LNT films. All of the scale bars unspecified represent $10 \mu \mathrm{m}$. (a) The upper and lower images on the left side are digital micrographs of typical GNP-included films having a width of approximately $10 \mu \mathrm{m}$, which have been mixed with 5-nm GNPs. From a fluorescence micrograph on the right side, we can see a partially fluorescent part on a border area of a GNP-included film stained by R6G fluorescent molecules. (b) While a digital microscopy image on the left side shows an LNT film mixed with 40-nm GNPs, a fluorescence micrograph on the right side exhibits a bright rectangle, which corresponds to an R6G-added film that has been mixed with 40-nm GNPs. (c) Despite the mixture of 5-nm GNPs, an R6G-added film having $30-\mu \mathrm{m}$ width provides a fluorescent area overall the film (the leftmost image), whereas the middle image observed using transmitted light displays the identical film and the digital microscopy magnifies the edge (the rightmost micrograph). (d) A schematic comparison between even and rough ends in capillary action of GNPs into LNT films. The sectional plane is more likely to be flat as the width $d$ is closer to the mean LNT length $L$, facilitating to capture GNPs as shown in the left schematic. The right diagram, on the other hand, illustrates that an irregular entrance formed by uneven terminations on the edge interferes with GNP-encapsulation into the inner space of individual LNTs. 
The contrasted results of Figures 3(a) and 3(b) are interpreted by the size-selectivity of LNT films; however, it remains to be proved that the FRET in LNT films actually requires the $10-\mu \mathrm{m}$ width, though the relation, $d<2 L$, should reduce serial arrays of LNTs in comparison with a wider film such that $d>2 L$. For making the ambiguity clear, we controlled another type of LNT film that was mixed with the identical 5-nm GNPs but was extended to $30 \mu \mathrm{m}$ in width (see the middle micrograph in Fig. 3(c)); the 5-nm GNPs could be inserted into LNT films irrespectively of the width, if the FRET mainly occurs due to unwrapped GNPs such as those existing in the interstices among LNTs. As shown in the left micrograph of Figure 3(c), the threefold width provides fluorescent films to the contrary. Furthermore, it is found from comparing fluorescent micrographs in Figures 3(a) to 3(c) that the fluorescent area in this $30-\mu \mathrm{m}$ film of Figure 3(c) does not cover a whole rectangle with its edge being obscure. In other words, the FRET seems to be relevant on edges of a $30-\mu \mathrm{m}$ film, which is consistent with a magnified view of this film in Figure 3(c) on the rightmost side where a metallic deposition of 5-nm GNPs is observed on the bordered region.

These results of Figure 3(c) imply the other significant factor of adjusting the width, which is schematically illustrated by Figure 3(d): the inner capacity of parallel LNTs is efficiently and homogeneously filled with 5-nm GNPs when the electrode gap length $d$, adjusted close to the mean LNT length $L$, creates a flat section as shown in the left diagram in Figure 3(d). For irregular ends, on the other hand, the inflow of 5-nm GNPs seems to be blocked out on the entrance region of open ends (see the right schematic in Fig. 3(d)), which explains the jamming of GNPs on film edges as shown in Figure 3(c).

A comparative study of the assembly courses. We need to validate that the off-chip assembly is mainly driven by dielectrophoresis for systematically searching an optimum set of electric parameters, such as the strength and frequency of the applied electric fields. To this end, we first investigate the assembly processes of carboxylatemodified polystyrene (CM-PS) spheres with their diameter of $3 \mu \mathrm{m}$, instead of LNTs. As shown in Figure 4(a), the off-chip assembly of $3-\mu \mathrm{m}$ spheres produces a hexagonal packing with twodimensional crystalline order, as do the on-chip techniques.

As soon as the external field is applied at $t=0$ via the electrode pair, the $3-\mu \mathrm{m}$ particles are attracted into the rectangular area, as depicted in Figure 1(b), and simultaneously we started to count the total number $N(t)$ of collected particles existing at a duration time $t$ in the rectangular region formed by the electrode pair, which has a 30$\mu \mathrm{m}$ gap and a $90-\mu \mathrm{m}$ width. Incidentally, we have set the maximum total number in Supplementary Figure S2(a) to be less than $N_{\max }=$ $7.4 \times 10^{2}$ because more electrically attracted colloids are localised around the electrode tips outside the rectangular region. As evaluated in Supplementary Discussion, $N_{\max }$ is equal to that for randomly close packed spheres ${ }^{51}$ in the box of an electric holder having a minimum volume $A_{\max } H_{\text {min }}$, with a prescribed rectangular area $A_{\max }=90 \mu \mathrm{m} \times 30 \mu \mathrm{m}=2700 \mu \mathrm{m}^{2}$ and a lower bound of mean height $H_{\min }=6.1 \mu \mathrm{m}$. The former area is calculated from the aforementioned electrode configuration, whereas the latter height $H_{\text {min }}$ reasonably explains the mean thicknesses of LNT films of several microns, which have been determined using the adjustment of the SEM focus when observing dry LNT films.

As shown in Supplementary Figure S2(a), the collected number, $N(t)$, as a function of the duration time $t$ is proportional to $t$ irrespective of the electric field strength in the range $0 \leq N(t) \leq N_{\max }$, and therefore the global slope of $N(t)$ uniquely determines the assembly number rate, $V_{N}=d N(t) / d t$, per second. Figure $4(\mathrm{~b})$ is a log-log plot of $V_{N}$ versus the field strength $E$ at $f=100 \mathrm{kHz}$, indicating that $V_{N} \sim E^{2}$ similarly to that of the dielectrophoretic velocity ${ }^{46,47,50}$. As explained in Supplementary Discussion, the $E$-dependence of $V_{N}$ proves the dielectrophoretic mechanism of the colloidal assembly in the plug-in system.
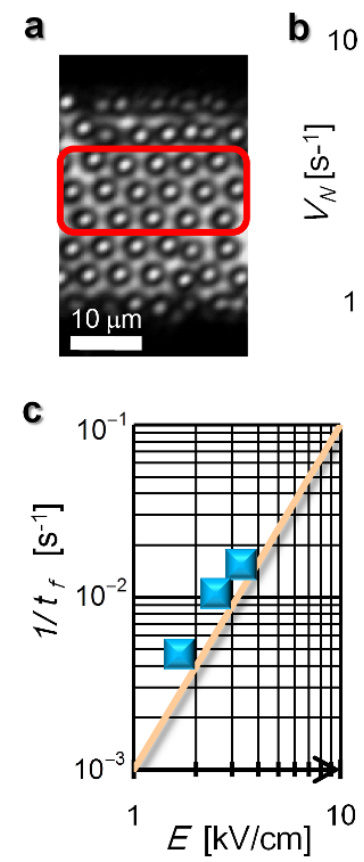

d

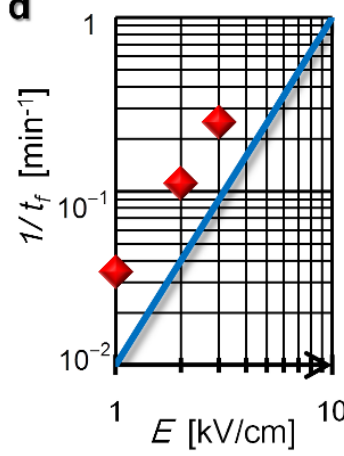

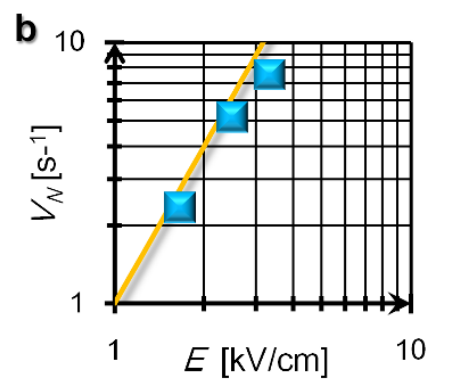

Figure 4 | Comparison between assembly rates of CM-PS particles and LNTs. (a) A magnified view of the first layer formed by CM-PS particles at $t=10$ seconds. We can see hexagonal packing within the marked area. (b) The assembly number rates $V_{N}$ of the spherical colloids as a function of E. A straight line representing the relation $V_{N} \sim E^{2}$ is shown for comparison. (c,d) Log-log plots of the inverse filling times $1 / t_{f}$ versus $E$ regarding either CM-PS particles (c) or LNTs (d). Straight lines in both (c) and (d), representing the relation $1 / t_{f} \sim E^{2}$, are shown for comparison.

However, turning our attention to the LNTs, we find it hard to count individual LNTs, so an alternative parameter to $N(t)$ needs to be explored. We then adopted the percentage fraction $P_{A}(t)$ of the cross-sectional area occupied by either CM-PS particles or electrically collected LNTs, where $P_{A}(t)$ is defined, using the relation $P_{A}(t)=$ $100\left(A(t) / A_{\max }\right)$, as the ratio of the measured area $A(t)$ to the maximum of $A_{\max }$. Tracing the evolution of an occupied area that covers both the inside and outside of the rectangular region, $P_{A}(t)$ increases to and crosses $100 \%$ at $t_{f}$ when colloidal particles with their number of $N\left(t_{f}\right)$ complete to fill the rectangular area prior to reaching the maximum state of $N_{\max }$ (see Supplementary Fig. S2(b)), and we have found that $N\left(t_{f}\right)$ of CM-PS particles is independent of $E$ as described in Supplementary Discussion. Because $V_{N}$ is given by $V_{N}=N\left(t_{f}\right) / t_{f}$ using an invariant number of $N\left(t_{f}\right)$, it follows that the inverse of $t_{f}$ obeys the $E$-dependence of $V_{N}$. Figure $4(c)$ actually demonstrates that $1 / t_{f} \propto E^{2}$ for CM-PS particles in accord with the relation of $V_{N}$.

We then measured $P_{A}(t)$ using the optical micrographs to evaluate $t_{f}$ of LNTs (Supplementary Fig. S5(a)). Figure 4(d) displays the log$\log$ plot of $1 / t_{f}$ versus $E$, where the experimental results satisfy the relation $1 / t_{f} \sim E^{2}$ as well as that for CM-PS particles. From Supplementary Figure S6, it is also found that the assembly degrees of CM-PS particles and LNTs are similarly reduced with an increase in $f$ in the range $100 \mathrm{kHz} \leq f \leq 700 \mathrm{kHZ}$, which corresponds to a typical $f$-dependence of dielectrophoresis ${ }^{52}$. Combining these consistent dependencies of the assembly courses of CM-PS particles and LNTs on $E$ and $f$, we conclude that dielectrophoresis causes the offchip assembly of LNTs and of colloidal spheres. Consequently, we fabricated the dry LNT films shown in Figures 2 and 3 using the electric parameters $E=3 \mathrm{kV} / \mathrm{cm}$ and $f=100 \mathrm{kHz}$, which have been optimised in terms of both the efficiency and robustness of the dielectrophoretic assembly.

The last parameter to be adjusted is the height $h$ from the substrate of the off-chip assembly in adopting an optimum set of $E=3 \mathrm{kV} / \mathrm{cm}$ 
and $f=100 \mathrm{kHz}$. As described in Supplementary Discussion using the results of Supplementary Figure S4, we chose $h=50 \mu \mathrm{m}$ for the fabrication of LNTs as a trade-off, considering the successful drying versus an efficient assembly or a sound removal of the electric mould.

\section{Discussion}

To summarise, the off-chip assembly method using plug-in electrodes has three properties: size adjustability of the potential valley, colloidal arrays lifted above the substrate, and electrode needles with tapered shapes. The first two advantages of this technique have been described in detail, and we add another benefit associated with the last feature below. The electrode tips produce the largest field gradient around them, thereby inducing colloidal dielectrophoresis mainly directed toward their pointed heads. When we place the needle tips of twin absorbers on opposite sides as shown in Figure 1(b), colloidal flows are generated from the outside to the inside across both sides of the rectangular edges vertical to the electrodes (see Supplementary Fig. S3). From the symmetrical pathways, it follows that gathering particles will meet in the middle region of the potential valley. Similar flows during plug-in assembly of LNTs work to exclude voided assembly films caused by bottlenecks during the initial stage.

Final remarks are made on the potentials as nano-assay or nanoreactor devices. Thanks to the reconfigurability, the off-chip assembly method is capable of providing a high-throughput nanofluidic device of LNTs that efficiently transports and reacts confined particles inside an LNT and that multiplies either the signalling or product of each LNT. Moreover, the scalability of an LNT film to a macroscopic length opens up the possibility for creating a variety of flexible nanofluidic devices that make chemical or biological reactions visible.

\section{Methods}

Materials. The lipid nanotubes (LNTs) used were derived from self-assembly in a glycolipid solution following a typical procedure, as described previously ${ }^{12}$. The inner diameters were $40 \pm 10 \mathrm{~nm}$, while the wall thicknesses were approximately $80 \mathrm{~nm}$. Because we set the electrode gap to $10 \mu \mathrm{m}$, the number of LNTs shorter than $5 \mu \mathrm{m}$, which are unfavourable particles, as described in the text (see also Figure 1(c)), needs to be diminished. The following recipe was therefore adopted to prepare the LNT solution. We first dissolved LNTs in deionised water to obtain a 0.1 -wt.\% dispersion, which was subsequently filtered through a $5-\mu \mathrm{m}$ plastic filter (17594K, Sartorius). Deionised water, 3-ml, was carefully added to the filter to redissolve the LNTs remaining on the filter. A $20-\mu \mathrm{l}$ aliquot of the filtered suspension was dropped onto a cover slip for electric assembly, yielding naturally dried LNT films.

The prepared LNT assembly was further dried in a vacuum to enhance the capillary action to fill the inner space of the LNTs with 5-nm GNPs. We induced capillary action by dropping a $20-\mu \mathrm{l}$ aliquot of a $0.1-$ wt. $\%$ GNP solution with 5 -nm diameters (Wako) onto a dried LNT film. Once again, we performed vacuum drying on the GNP-added films, which had subsequently been rinsed with an excess amount of 20wt.\% saline to remove GNPs from the outer surfaces of the LNTs. To observe the FRET, we added a 0.1-mM solution of Rhodamine 6G (R6G, Wako) molecules to the film with encapsulated 5-nm GNPs. As a control experiment, we also used a 0.1-wt.\% solution of GNPs having a larger diameter of $40 \mathrm{~nm}$ (Wako).

For a reference study of the electric assembly process, we investigated the gathering courses of carboxylate-modified polystyrene (CM-PS) particles $3-\mu \mathrm{m}$ in diameter (Dainichi-Seika) in a $100-\mu \mathrm{l}$ aliquot of a $0.1 \mathrm{wt} \%$ suspension.

Experimental setup. We mounted an aliquot on an inverted fluorescence microscope (Nikon, TE2000-U), into which a pair of tungsten microelectrodes with tip diameters of $1 \mu \mathrm{m}$ was inserted. An external electric field having a sinusoidal wave was applied between the electrode pair using an arbitrary waveform generator (Agilent, 33220A) and a current amplifier (FLC Electronics, F30PV), and the microscopy images and movies were obtained using a CCD camera (Q-Imaging, Retiga Exi). We performed video analysis using image analysis software (ImageJ). The positions of the electrode needles are independently controlled using two sets of patch-clamp micromanipulators (Narisige, NMN-21). Observations of prepared LNT films were also made using a digital microscope (Omron, VC7700), a polarised microscope (Olympus, BX51), and a field emission scanning electron microscope (JEOL, JSM$7300 \mathrm{~F})$ at $3 \mathrm{kV}$.

1. Xia, Y., Gates, B., Yin, Y. \& Lu, Y. Monodispersed colloidal spheres: old materials with new applications. Adv. Mater. 12, 693-713 (2000).
2. Li, F., Josephson, D. P. \& Stein, A. Colloidal assembly: the road from particles to colloidal molecules and crystals. Angew. Chem. Int. Ed. 50, 360-388 (2011).

3. Grzelczak, M., Vermant, J., Furst, E. M. \& Liz-Marzan, L. M. Directed selfassembly of nanoparticles. ACS Nano 4, 3591-3605 (2010)

4. Velev, O. D. \& Gupta, S. Materials fabricated by micro- and nanoparticle assembly: the challenging path from science to engineering. Adv. Mater. 21, 1897-1905 (2009).

5. Yethiraj, A. Tunable colloids: control of colloidal phase transitions with tunable interactions. Soft Matter 3, 1099-1115 (2007).

6. van Blaaderen, A. Colloids under external control. MRS Bull. 25, 85-90 (2004).

7. Holtz, J. H. \& Asher, S. A. Polymerized colloidal crystal hydrogel films as intelligent chemical sensing materials. Nature 389, 829-832 (1997).

8. Schnur, J. M. Lipid tubules: a paradigm for molecularly engineered structures. Science 262, 1669-1676 (1993).

9. Zhang, S. Fabrication of novel biomaterials through molecular self-assembly. Nat. Biotechnol. 21, 1171-1178 (2003).

10. Karlsson, A. et al. Molecular engineering: networks of nanotubes and containers. Nature 409, 150-152 (2001).

11. Jesorka, A. et al. Generation of phospholipid vesicle-nanotube networks and transport of molecules therein. Nat. Protoc. 6, 791-805 (2011).

12. Kamiya, S. et al. Molecular structure of glucopyranosylamide lipid and nanotube morphology. Langmuir 21, 743-750 (2005).

13. Shimizu, T. Self-assembled lipid nanotube hosts: the dimension control for encapsulation of nanometer-scale guest substances. J. Polym. Sci. A1 44, 5137-5152 (2006).

14. Shimizu, T. Self-assembled organic nanotubes: toward attoliter chemistry. J. Polym. Sci. A1 46, 2601-2611 (2008).

15. Zhou, Y. \& Shimizu, T. Lipid nanotubes: a unique template to create diverse onedimensional nanostructures. Chem. Mater. 20, 625-633 (2008).

16. Kameta, N., Minamikawa, H. \& Masuda, M. Supramolecular organic nanotubes: how to utilize the inner nanospace and the outer space. Soft Matter 7, 4539-4561 (2011).

17. Martin, C. R. \& Kohli, P. The emerging field of nanotube biotechnology. Nat. Rev. Drug Discov. 2, 29-37 (2003).

18. Wakasugi, A. et al. Organic nanotubes for drug loading and cellular delivery. Int. J. Pharm. 413, 271-278 (2011).

19. Ding, W. et al. Hybrid organic nanotubes with dual functionalities localized on cylindrical nanochannels control the release of doxorubicin. Adv. Healthc. Mater. 1, 699-706 (2012).

20. Lo, P. K. et al. Loading and selective release of cargo in DNA nanotubes with longitudinal variation. Nat. Chem. 2, 319-328 (2010).

21. Losic, D. \& Simovic, S. Self-ordered nanopore and nanotube platforms for drug delivery applications. Expert Opin. Drug Del. 6, 1363-1381 (2009).

22. Kameta, N., Masuda, M. \& Shimizu, T. Soft nanotube hydrogels functioning as artificial chaperones. ACS Nano 6, 5249-5258 (2012).

23. Kameta, N. et al. Functionalizable organic nanochannels based on lipid nanotubes: encapsulation and nanofluidic behavior of biomacromolecules. Chem Mater. 19, 3553-3560 (2007).

24. Karlsson, M. et al. Biomimetic nanoscale reactors and networks. Annu. Rev. Phys. Chem. 55, 613-649 (2004).

25. Sparreboom, W., van den Berg, A. \& Eijkel, J. C. T. Principles and applications of nanofluidic transport. Nat. Nanotechnol. 4, 713-720 (2009).

26. Fraikin, J.-L., Tambet, T., McKenney, C. M., Ruoslahti, E. \& Cleland, A. N. A highthroughput label-free nanoparticle analyser. Nat. Nanotechnol. 6, 308-313 (2011)

27. Song, H., Chen, D. L. \& Ismagilov, R. F. Reactions in droplets in microfluidic channels. Angew. Chem. Int. Ed. 45, 7336-7356 (2006).

28. Arora, A., Simone, G., Salieb-Beugelaar, G. B., Kim, J. T. \& Manz, A. Latest developments in micro total analysis systems. Anal. Chem. 82, 4830-4847 (2010).

29. Frusawa, H. et al. Aligning a Single-Lipid Nanotube with Moderate Stiffness. Angew. Chem. Int. Ed. 115, 76-78 (2003).

30. Guo, Y. et al. Alignment of glycolipid nanotubes on a planar glass substrate using a two-step microextrusion technique. J. Nanosci. Nanotechno. 6, 1464-1466 (2006).

31. Fujima, T., Frusawa, H., Minamikawa, H., Ito, K. \& T Shimizu, T. Elastic precursor of the transformation from glycolipid nanotube to vesicle. J. Phys.-Condens. Mat. 18, 3089 (2006).

32. Nogawa, K. et al. Development of novel nanopipette with a lipid nanotube as nanochannel. J. Robot. Mechatron. 19, 528-534 (2007).

33. Hirano, K. et al. Measuring the length distribution of self-assembled lipid nanotubes by orientation control with a high-frequency alternating current electric field in aqueous solutions. Anal. Chem. 81, 1459-1464 (2009).

34. Mahajan, N. \& Fang, J. Two-dimensional ordered arrays of aligned lipid tubules on substrates with microfluidic networks. Langmuir 21, 3153-3157 (2005).

35. Zhao, Y. \& Fang, J. Positioning and alignment of lipid tubules on patterned Au substrates. Langmuir 22, 1891-1895 (2006).

36. Fang, J. Ordered arrays of self-assembled lipid tubules: fabrication and applications. J. Mater. Chem. 17, 3479-3484 (2007).

37. Masuda, H. \& Fukuda, K. Ordered metal nanohole arrays made by a two-step replication of honeycomb structures of anodic alumina. Science 268, 1466-1468 (1995)

38. Li, L., Koshizaki, N. \& Li, G. Nanotube arrays in porous anodic alumina membranes. J. Mater. Sci. Tech. 24 (2008). 
39. Losic, D., Velleman, L., Kant, K., Kumeria, T., Gulati, K., Shapter, J. G., Beattie, D. A. \& Simovic, S. Self-ordering electrochemistry: a simple approach for engineering nanopore and nanotube arrays for emerging applications. Aust. J. Chem. 64, 294-301 (2011).

40. Tan, L. H. et al. Fabrication of polymer nanocavities with tailored openings. ACS Nano. 24, 3469-3474 (2009).

41. Yaman, M. et al. Arrays of indefinitely long uniform nanowires and nanotubes. Nat. Mater. 10, 494-501 (2011).

42. Shekhar, S., Stokes, P. \& Khondaker, S. I. Ultrahigh density alignment of carbon nanotube arrays by dielectrophoresis. ACS Nano 5, 1739-1746 (2011).

43. Sarker, B. K., Shekhar, S. \& Khondaker, S. I. Semiconducting enriched carbon nanotube aligned arrays of tunable density and their electrical transport properties. ACS Nano 5, 6297-6305 (2011).

44. Hermanson, K. D., Lumsdon, S. O., Williams, J. P., Kaler, E. W. \& Velev, O. D Dielectrophoretic assembly of electrically functional microwires from nanoparticle suspensions. Science 294, 1082-1086 (2001).

45. Velev, O. D. \& Bhatt, K. H. On-chip micromanipulation and assembly of colloidal particles by electric fields. Soft Matter 2, 738-750 (2006).

46. Sullivan, M. T. et al. An electric bottle for colloids. Phys. Rev. Lett. 96, 015703 (2006).

47. Leunissen, M. E., Sullivan, M. T., Chaikin, P. M. \& van Blaaderen, A. Concentrating colloids with electric field gradients. I. Particle transport and growth mechanism of hard-sphere-like crystals in an electric bottle. J. Chem. Phys. 128, 164508 (2008).

48. Juarez, J. J., Feicht, S. E. \& Bevan, M. A. Electric field mediated assembly of three dimensional equilibrium colloidal crystals. Soft Matter 8, 94-103 (2012).

49. Mittal, M. \& Furst, E. M. Electric field-directed convective assembly of ellipsoidal colloidal particles to create optically and mechanically anisotropic thin films. $A d v$. Funct. Mater. 19, 3271-3278 (2009).
50. Voldman, J. Electrical forces for microscale cell manipulation. Annu. Rev. Biomed. Eng. 8, 425-454 (2006).

51. Parisi, G. \& Zamponi, F. Mean-field theory of hard sphere glasses and jamming Rev. Mod. Phys. 82, 789-845 (2010).

52. Morgan, H., Hughes, M. P. \& Green, N. G. Separation of submicron bioparticles by dielectrophoresis. Biophys. J. 77, 516-525 (1999).

\section{Author contributions}

H.F. designed the experiments, analyzed the data, and wrote the manuscript. T.M. performed the experiments regarding lipid nanotubes (LNTs) and contributed to the data analysis. E.K. measured the assembly processes of spherical colloids and contributed to the data analysis. K.H. conducted the length measurements of LNTs and analyzed the data. N.K. and M.M. synthesized LNTs, observed the LNT films using polarised optical microscopy, discussed all of the experimental results, and reviewed the manuscript. T.S. supervised the project and commented on the manuscript.

\section{Additional information}

Supplementary information accompanies this paper at http://www.nature.com/ scientificreports

Competing financial interests: The authors declare no competing financial interests.

How to cite this article: Frusawa, H. et al. Electric moulding of dispersed lipid nanotubes into a nanofluidic device. Sci. Rep. 3, 2165; DOI:10.1038/srep02165 (2013).

(c) (1) () $\odot$ This work is licensed under a Creative Commons Attribution-

BY NG ND NonCommercial-NoDerivs 3.0 Unported license. To view a copy of this license, visit http://creativecommons.org/licenses/by-nc-nd/3.0 